\title{
Caring for BRCA Carriers: Strategies to Promote Health and Preserve Fertility
}

\author{
Nola S. Herlihy ${ }^{1,2, *}$, Lucky Sekhon ${ }^{1,2}$, Joseph A. Lee ${ }^{1}$, Daniel Stein ${ }^{1,2}$, Alan B. Copperman ${ }^{1,2}$, Matthew A. \\ Lederman $^{1,2}$
}

${ }^{1}$ Reproductive Medicine Associates of New York, 635 Madison Ave 10 ${ }^{\text {th }}$ Floor, New York, New York, United States, 10022

${ }^{2}$ Department of Obstetrics, Gynecology and Reproductive Science, Icahn School of Medicine at Mount Sinai, 1176 Fifth Avenue, 9th Floor, New York, New York, United States, 10029

${ }^{*}$ Corresponding author: Nola Herlihy, Reproductive Medicine Associates of New York, 635 Madison Ave $10^{\text {th }}$ Floor, New York, New York, United States, 10022; Tel: 404-862-5100; E-mail: Nola.herlihy@mountsinai.org

Received Date: February 05, 2018; Accepted Date: March 02, 2018; Published Date: March 08, 2018

Citation: Nola S. Herlihy (2018) Caring for BRCA Carriers: Strategies to Promote Health and Preserve Fertility. J Womens Health Gyn 5: 1-10.

\begin{abstract}
BRCA1 and BRCA2 mutation carriers are at increased risk for breast and ovarian cancer, with lifetime risks approximately $49-72 \%$ for breast cancer and $17-59 \%$ for ovarian cancer. The National Cancer Comprehensive Network recommends routine screening as well as a risk-reducing surgery to remove the fallopian tubes and ovaries for BRCA carriers between ages 35 to 40 or after the completion of childbearing. Since many women have not completed childbearing by age 40 , they have the option of undergoing fertility preservation prior to undergoing this risk-reducing surgery. Additionally, use of Preimplantation Genetic Diagnosis (PGD) allows couples to prevent transmission to their offspring. BRCA carriers may be at greater risk for diminished ovarian reserve, yet studies regarding the effect of BRCA carrier status on fertility remain inconsistent in their conclusions. To date, researchers have demonstrated that infertility treatment is safe in BRCA carriers. BRCA carriers are faced with complex challenges and will benefit from consultation with a fertility specialist to discuss options for fertility preservation to safely build the family they desire.
\end{abstract}

Keywords: Breast neoplasms; Ovarian neoplasms; Fertility; Cryopreservation; Preimplantation genetic diagnosis

\section{Introduction}

The majority of breast and ovarian cancers result from sporadic mutations. Of women with breast cancer, approximately $15 \%$ will have at least one affected relative, demonstrating that a minority are due to hereditary cancer syndromes [1]. BRCA1 and BRCA2 germline mutations are understood to influence up to $20 \%$ of hereditary breast and ovarian cancers, $10 \%$ of ovarian and $3-5 \%$ of breast cancer $[2,4]$. BRCA is estimated to be $0.33-0.13 \%$ of the general population [5] with the highest prevalence in the Ashkenazi Jewish community (3\%) [6]. The BRCA genes are tumor suppressor genes, which encode proteins responsible for DNA mismatch repair. BRCA carriers are susceptible to an accumulation of damaged DNA that could contribute to increased cancer risk and earlier onset of cancer development [6]. BRCA carriers have a lifetime-estimated risk of breast (49-72\%) and ovarian (17-59\%) cancer (table 1) [7-9].

(C)2018 The Authors. Published by the JScholar under the terms of the Creative Commons Attribution License http://creativecommons.org/licenses/by/3.0/, which permits unrestricted use, provided the original author and source are credited.
Vigilant screening recommendations are standard. Often, risk-reducing surgery (i.e. removal of the fallopian tubes and ovaries) is performed after the conclusion of family building in affected womenor between age 35-40 [10]. Given that many patients have not yet completed child bearing by this age, fertility preservation through cryopreservation of oocytes and/ or embryos should be considered prior to surgery. Clinicians must convey realistic expectations regarding patient prognosis, as BRCA carriers may be at risk for diminished ovarian reserve (DOR). This clinical perspective summarizes breast and ovarian cancer preventative strategies, tours fertility preservation and family building options, discusses potential BRCA and DOR association, and explores ovarian stimulation in BRCA carriers. 


\begin{tabular}{|l|l|l|}
\hline & $\begin{array}{l}\text { Lifetime Breast } \\
\text { Cancer Risk }\end{array}$ & $\begin{array}{l}\text { Lifetime Ovarian } \\
\text { Cancer Risk }\end{array}$ \\
\hline BRCA 1 & $57-72 \%$ & $40-59 \%$ \\
\hline BRCA 2 & $49-69 \%$ & $17-18 \%$ \\
\hline $\begin{array}{l}\text { General Popu- } \\
\text { lation }\end{array}$ & $12 \%$ & $1.4 \%$ \\
\hline
\end{tabular}

Table I.Risk of cancer in BRCA patients [7-9].

\section{Cancer Prevention: Screening Strategies and use of Pro-}

\section{phylactic Surgery}

Current guidelines for breast cancer screening in BRCA carriers recommend clinical breast exams every 6 months and annual MRI or mammogram beginning at age 25 - or sooner - depending on the earliest age of onset in the family [11]. A combination of clinical breast exam, mammography and MRI has the highest sensitivity for detecting breast cancer [12-13].

Prophylactic surgery and chemoprevention are two ways to reduce the risk of breast cancer development. According to the National Comprehensive Cancer Network (NCCN) guidelines, women with BRCA mutations may be offered prophylactic mastectomy. Prophylactic bilateral total mastectomy has shown to reduce the rate of breast cancer by $92 \%$ and related deaths by $81 \%$ in healthy BRCA carriers [14,15]. A large prospective multi-center cohort study of 2,482 women with a known BRCA mutation demonstrated that $100 \%$ of women who underwent risk-reducing mastectomy did not develop breast cancer while $7 \%$ of those who did not have surgery developed cancer within the 35 year span of the study [16]. Although its efficacy and safety requires further research, Tamoxifen, a selective estrogen-receptor modulator (SERM), has shown to be a promising chemoprevention agent to prevent and/or treat breast cancer.

Prevention strategies for ovarian cancer are similar to those for breast cancer, including early surveillance and prophylactic surgery. NCCN guidelines advise clinicians to consider concurrent transvaginal ultrasound and CA-125 every six months beginning at age 30 , or 5 to 10 years before the earliest onset of a family diagnosis of ovarian cancer [11]. However, screening appears to have limited benefit as it has not been shown to decrease mortality, especially with high-risk patients. Chemoprevention with the use of oral contraceptive pills (OCPs) is a plausible consideration, as OCPs have been shown to decrease the risk of ovarian cancer in BRCA carriers and the general population. However, the risk of ovarian cancer remains high, and BRCA carriers may for go the use of OCPs due to the potential increased risk of breast cancer [17]. Due to limited options for prevention, risk-reducing bilateral salpingo-oophorectomy (BSO) is advised at age 35-40 or after childbearing completion. A BSO reduces the risk of ovarian and primary peritoneal cancer by $80 \%$ in BRCA carriers [18]. Mounting evidence demonstrates that ovarian cancer originates in the fallopian tubes, hence, interval salpingectomy with delayed oophorectomy (ISDO) has been suggested for women wishing to delay oophorectomy [19].
Kwon et al performed a cost-benefit analysis of BSO, bilateral salpingectomy and ISDO, and found that $\mathrm{BSO}$ was associated with lowest cost and highest life expectancy, but ISDO yielded the highest quality-adjusted life expectancy [20]. However, the use of ISDO increases the potential for surgical complications as it requires two, separate surgical procedures. Given that the protective benefit of ISDO is still uncertain and the need for two procedures may lead to decreased compliance, the technique is not the standard of care and should only be considered for women who decline oophorectomy. Surgicallyinduced premature menopause carries its own health consequences including vasomotor symptoms and decreased sexual function as well as increased risk for cardiovascular disease and osteoporosis. BRCA carriers without a history of breast cancer should consider hormone replacement therapy (HRT) after surgery to mitigate these effects; HRT has not been associated with increased risk of breast cancer in this population [21].

\section{BRCA and Infertility}

The pathophysiologic mechanisms connecting BRCA mutations and infertility are not well understood, however a variety of links have been proposed. BRCA mutations may affect ovarian function by decreasing ovarian reserve, defined as oocyte quantity, quality, and reproductive potential [22]. BRCA1 and BRCA2 are part of the family of ataxia-telangiectasia-mutated (ATM)-mediated DNA double strand repair genes, and have critical roles in the DNA repair pathway. Inefficient repair causes DNA damage accumulation and contributes to oocyte aging, apoptosis, and depletion [23,24]. Titus et al. published findings in which BRCA1 heterozygous mutant mice produced fewer oocytes in response to ovarian stimulation and had smaller litter sizes than wild-type cohorts. Furthermore, the total primordial follicle numbers per mouse ovary were lower and a significantly higher percentage of follicles accumulated DNA damage as a result of deficient double stranded break repair [25].

BRCA mutations have been postulated to influenceoocyte rates of aneuploidy. Primarily oocytes utilize DNA repair mechanisms while undergoing homologous recombination prior to meiotic arrest. Failure to repair the double stranded DNA correctly leads to deletions, translocations, and chromosome loss [26,27]. A mouse model study demonstrated that BRCA1 is required for meiotic spindle assembly and checkpoint activation, for which any disruption could lead to aneuploidy [28]. There is limited data on the risk of embryo aneuploidy in BRCA carriers; however, one IVF study demonstrated no significant difference in the rate of aneuploidy in BRCA 1 and 2 carriers [29]. BRCA1 is further known to play a role in maintaining genome integrity and mutations are associated with reduced cell proliferation and impaired embryogenesis $[23,30,31]$. To date, there are no published studies comparing the incidence of embryonic aneuploidy among BRCA carriers and non-BRCA control patients undergoing IVF. 


\section{Diminished Ovarian Reserve in BRCA Carriers}

The literature remains inconclusive regarding the potential links between BRCA gene mutations and decreased ovarian reserve, but many studies suggest a direct relationship. Reduced parity, often observed in BRCA carriers, may reflectDOR in these patients [32]. However, parity is not a reliable marker of ovarian reserve as it could be influenced by patient preferences for family size, apprehension for BRCA heredity, and use of risk-reducing BSO. Additionally, two studies have demonstrated early onset of natural menopause in BRCA mutation carriers. A cohort comparing 382 BRCA carriers to 765 non-carriers showed a significantly younger mean age of menopause ( 50 vs 53 years, $\mathrm{p}<0.001$ ) [33]. Finch et al. similarly displayed a decrease of 2 years in menopause for BRCA1 carriers and a decrease of a year in BRCA2 carriers in a case control of 908 matched pairs [34]. Several studies examining the association between anti-Müllerian hormone (AMH) levels, a well-known marker of ovarian reserve, and BRCA carrier status show conflicting data. A small study of 41 healthy BRCA carriers failed to demonstrate a statistically significant difference in AMH levels compared to the general population [35]. Two studies have suggested that the BRCA1 mutation maybe implicated in DOR to a greater degree than the BRCA2 mutation. A study of 143 healthy reproductive-age women stratified BRCA1 and BRCA2 carriers and found, after controlling for age and BMI, that BRCA1 carriers have a significant decrease in serum AMH levels as compared to non-carriers $(0.53 \mathrm{ng} / \mathrm{mL}$ [95\% confidence interval (CI) $0.33-0.77 \mathrm{ng} / \mathrm{mL}]$ vs. $1.05[95 \%$ CI $0.76-1.40 \mathrm{ng} / \mathrm{mL}])$. BRCA2 carriers showed no difference in AMH levels as compared to non-carriers [36]. Philips et al. corroborated these findings in a cross sectional study of 693 women with BRCA1 or BRCA2 without a personal history of cancer. On average, BRCA1 carriers had 25\% (95\% CI: 5\%$41 \%, \mathrm{P}<0.02)$ lower AMH concentrations than non-carriers; and BRCA2 status, once again, was shown to have no effect on AMH level [37]. However, when comparing BRCA carriers and women at high risk for hereditary breast and ovarian cancer who are BRCA negative to low risk women, Johnson et al found that BRCA2 carriers and BRCA negative women have lower AMH levels than low risk women [38].

Three studies focused on DOR and ovarian response to IVF in BRCA carriers. In a study of 12 young breast cancer patients undergoing controlled ovarian hyperstimulation with co-administration of gonadotropins and letrozole, BRCA1 carriers demonstrated significantly reduced oocyte yield compared to controls and a third of carriers had low ovarian response [39]. Low ovarian response is largely accepted as less than four oocytes at vaginal oocyte retrieval (VOR) [39]. Notably there was no significant difference in ovarian response or oocyte yield between BRCA2 carriers and non-carriers. Derks-Smeets et al confirmed these findings in a retrospective study of 38 BRCA carriers and 154 controls, with multiple linear regression analysis revealing significantly lower yield of mature oocytes in BRCA1 patients [40]. However, a larger case-controlled study of 62 BRCA mutation carriers and 62 matched controls reported similar oocyte yields at VOR (13.75 vs. 14.75) and similar rates of low ovarian response among the patient cohorts $(8.06 \%$ vs. $6.45 \%)$ [ 41$]$.
More recently, Lin et al made a compelling argument for diminished ovarian reserve in BRCA carriers by demonstrating lower primordial follicle densities and higher DNA doublestranded breaks in primordial follicle oocytes in ovarian tissue samples from BRCA carriers compared to age matched controls [42].

\section{Options for Fertility Preservation}

Healthy BRCA mutation carriers are faced with several factors that may influence their decisions to conceive and include:

- If, and when, to undergo risk-reducing surgery, par ticularly the removal of the fallopian tubes and ovaries

- The potential risk of diminished ovarian reserve

- $\quad$ Age, which plays a role in all women's fertility

- Marital status

- $\quad$ Desired family size

- $\quad$ The risk for passing on the mutation to their off spring (as high as a $50 \%$ chance)

Although adoption and the use of gametes from unaffected donors in third party reproduction are available avenues to parenthood, most BRCA carriers prefer biological offspring $[43,44]$. Therefore, counseling patients on their reproductive options and potential impact on well-being is crucial.

BRCA carriers have options for family building. They may try to conceive naturally and elect for genetic testing of their offspring, such as prenatal diagnosis (PND), to prevent the transmission of the BRCA mutation. Alternatively, women have the option to utilize controlled ovarian hyperstimulation $(\mathrm{COH})$ followed by oocyte or embryo cryopreservation and preimplantation genetic diagnosis (PGD). With cryopreservation techniques, BRCA carriers who desire to extend their fertility can preserve it prior to undergoing risk-reducing surgery.

Given the modern improvements in cryopreservation technique, thousands of women have conceived using vitrified, thawed, fertilized, and transferred oocytes. As demonstrated by studies finding no increase in chromosomal abnormalities, birth defects, or developmental deficits in the offspring, oocytecryo preservation is considered a safe laboratory technique [45-47]. The improved efficacy of oocyte cryopreservation is further evidenced by the release of the American Society for Reproductive Medicine (ASRM) committee opinion in 2013, which states oocyte vitrification and warming "should no longer be considered experimental" [48].

Alternatively, retrieved oocytes can be fertilized and frozen as embryos. One of the advantages of freezing embryos is that they can be biopsied prior to freezing, which allows for PGD to test for the BRCA mutation and preimplantation genetic screening (PGS) to test for embryonic aneuploidy. With PGD, trophectoderm cells from embryos cultured during in vitro fertilization (IVF) are biopsied, and a genetic analysis screens embryos for the BRCA mutation. By selecting unaffected, healthy embryos, clinicians may assist patients in preventing transmission of the BRCA mutation to their offspring [49-51]. 
Focus group data demonstrated that BRCA carriers are interested in fertility preservation but feel health care providers do a suboptimal job of discussing these options and concerns [52]. Some of these patients require greater familiarity in the reproductive clinical impact of a risk reducing BSO on subsequent fertility or the benefit of fertility preservation consultation (i.e. oocyte or embryo banking) before undergoing a risk-reducing BSO [53]. Furthermore, most of these women have voiced tremendous emotional distress and worry about passing on the mutation to their offspring [54-57]. Most couples consider termination of an affected fetus as unacceptable, and favor PGD over PND as a means of BRCA prevention [58]. The use of PGD for the detection of BRCA mutations raises potential ethical concerns and highlights the issue of parental autonomy in reproductive decision-making [59-61]. Attitudes towards PGD are mixed, but most women surveyed believe PGD should be offered as part of clinical care and the ethical concerns are outweighed by the potential benefit of rearing healthy offspring(s) [43]. A study of couples with a known BRCA mutation who used PGD cited protection of their future children from the physical and psychological effects of BRCA as their primary motivation [58]. Furthermore, decisions are dictated by patients' own experiences with disease; those with a personal history of cancer and greater severity of disease are more likely to consider using PGD [62]. Similarly, nearly half of surveyed gynecologists and gynecologic oncologists in the United States believe PGD to be "appropriate" in the detection of cancer predisposition due to BRCA [63]. Despite these findings, data continues to highlight a lack of knowledge in BRCA carriers on fertility preservation options $[53,64]$. Therefore, all patients determined to be a BRCA carrier would seem to benefit from a consultation with a reproductive specialist prior to undergoing medical or surgical management [65-67].

Fertility specialists can apply treatment algorithms for BRCA mutation carriers who have not yet completed childbearing based on their age, relationship status and whether they plan to utilize PGD (Figure 1 and 2). BRCA carriers have the option to undergo oocyte cryopreservation at any age, and should strongly be encouraged to consider this option if they are over the age of 35 . Younger women under the age of 35, who are not yet ready to commit to having oocytes cryopreserved, may elect for annual monitoring of ovarian reserve (serum AMH level and transvaginal sonogram to assess a follicle count), with the goal of pursuing oocyte cryopreservation before any significant decline in ovarian reserve. Those with cryopreserved oocytes have the option of also undergoing PGD in the future.

BRCA carriers who plan to utilize PGD in the future to prevent transmission of BRCA to their offspring should be counseled by an IVF specialist as early as possible to maximize the number of embryos available for genetic screening and banking. Women undergoing PGD tend to also undergo aneuploidy screening simultaneously (PGS, preimplantation genetic screening, or CCS, comprehensive chromosomal screening). Aneuploidy is the leading cause of implantation failure and miscarriage after IVF [68-69].
The rate of aneuploidy increases with increasing maternal age, which explains why advanced-age women have lower pregnancy rates and higher miscarriage rates in an IVF cycle utilizing unscreened embryos. PGD and PGS allow for the identification of euploid, BRCA negative embryos which can be preserved through vitrification for future attempts at pregnancy. By understanding the female-age association to aneuploidy, couples committed to undergoing PGD to prevent BRCA transmission to their offspring should pursue fertility preservation treatment as early as possible to maximize euploid embryo yield. This strategy creates the most flexible plan to build a healthy family.

PGD allows for the selection and transfer of unaffected embryos. However, clinical and moral dilemmas arise when all the embryos are affected with the BRCA mutation or are indeterminate. In situations when the couple only has affected embryos, some couples may elect not to transfer, if the concern for future offspring with BRCA outweighs the desire for biological children. Others may consider transfer of only male embryos, with the knowledge that males are less likely to develop cancer as compared to females. One couple reported that the process of PGD only strengthened their desire to become parents, and elected to conceive naturally when presented with all affected embryos [58]. A case report of a patient who requested to transfer embryos of indeterminate status concluded that although this practice challenges the physician's duty to non-maleficence and social justice, transferring BRCA affected embryos was ethically permissible with adequate counseling [70]. In these circumstances, individualized care may be dictated by patient autonomy and guided by careful counseling with a geneticist and institutional ethics committees.

\section{Risks of Controlled Ovarian Hyperstimulation in BRCA}

\section{Carriers}

Women without a BRCA mutation are not more likely to develop breast cancer after the use of ART treatment. Zreik et al. performed a meta-analysis summarizing the results of 8 cohort and 15 case-control studies that examined the risk of breast cancer in patients taking fertility medications. They found no significant increased risk of breast cancer after taking both clomiphene citrate (RR 1.08 995\% CI 0.98-1.19) and composite gonadotropins (RR 0.99 (95\% CI 0.89-1.11). Furthermore, they found no significant risk of breast cancer associated with the number of completed treatment cycles (RR 1.08 (CI 0.92-1.26)) [71]. Belt-Dusebout et al. assessed the long-term risk of breast cancer after ovarian stimulation and observed that after a median follow up of 21 years, breast cancer risk in IVF-treated women was still not statistically different from that in the general population (standardized incidence ratio 1.01 [95\% CI, 0.93-1.09]) [72]. 


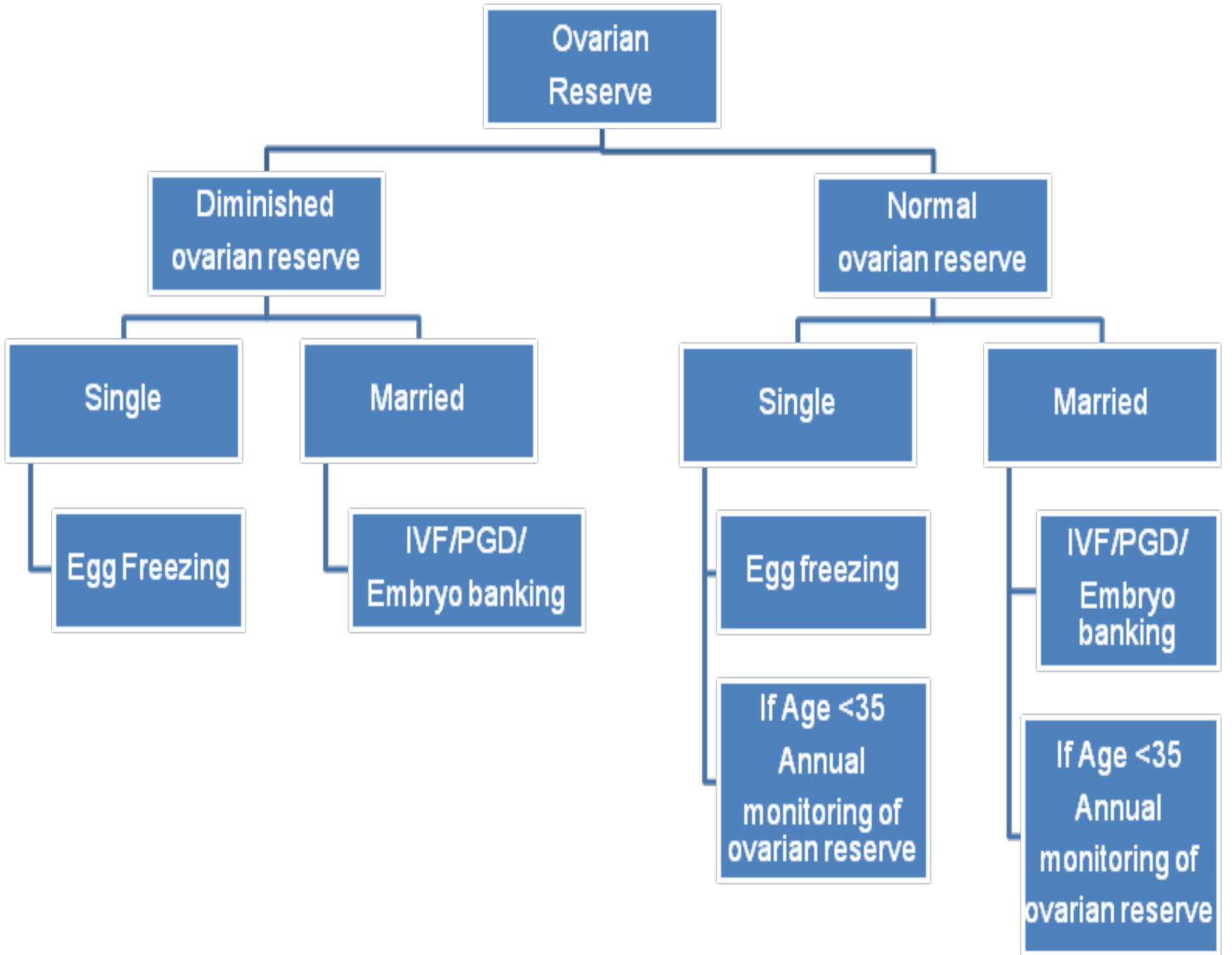

Figure 1: Options for BRCA carriers who plan to utilize PGD

This algorithm may be used to manage fertility preservation options for BRCA carriers who plan to use PGD to identify affected embryos and avoid transfer of BRCA mutation to offspring. 


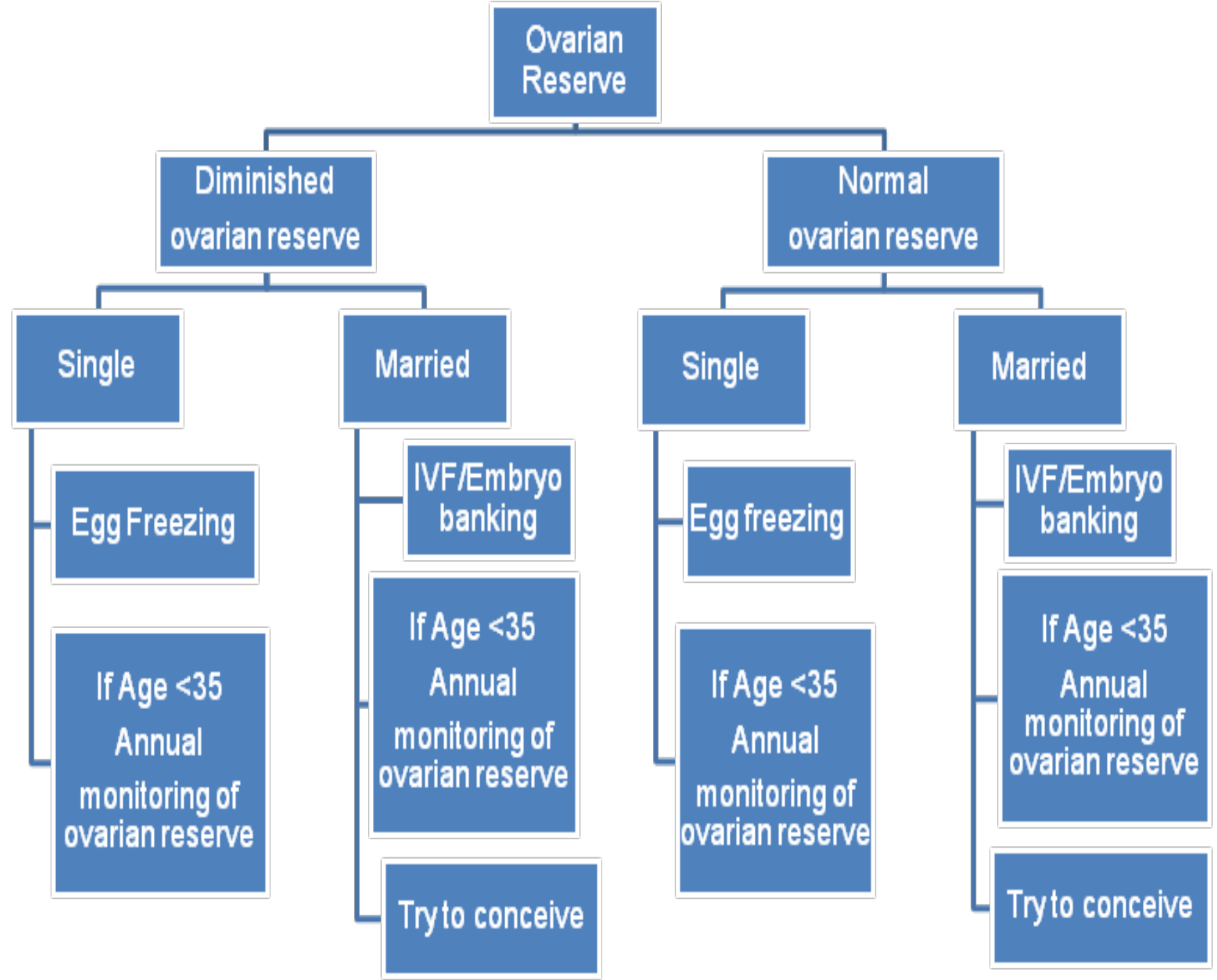

Figure 2: Options for BRCA carriers who do not plan to utilize PGD

This algorithm may be used to manage fertility preservation options for BRCA carriers who elect not to utilize PGD. 
BRCA carriers are predisposed to increased risk of developing breast cancer, but current literature does not demonstrate that undergoing $\mathrm{COH}$ further increases this risk. Kotsopoulos et al. conducted a matched case-control study of 1,380 pairs of women and found that BRCA carriers who had used fertility medications were not at increased risk of breast cancer (OR1.21, 95\% CI 0.81-1.82) compared to non-users [73]. A study of 337 women with breast cancer by Kim et al. compared outcomes of patients who underwent $\mathrm{COH}$ with coadministration of gonadotropins and letrozole prior to breast cancer treatment compared to patients who did not undergo any fertility preservation procedure and found no increased risk of recurrence after five years of follow-up and no effect on survival outcomes of BRCA carriers [74].

Previous studies have also examined the effect of fertility treatments on subsequent ovarian cancer risk within the general population. Rizzuto et al. performed a meta-analysis including 11 case control and 14 cohort studies and reported no increased incidence of invasive ovarian cancer among women undergoing ART treatment with any fertility medication [75]. These findings agreed with an earlier cohort of over 2,400 Israeli women who were followed for $>30$ years after exposure to infertility medication [76]. Previous studies that reported a positive association between infertility treatment and invasive ovarian cancer were limited by small sample sizes, imprecise drug exposure information and treatment indication, and short term follow up [77-79].

The risk of ovarian cancer in BRCA carriers undergoing infertility treatment is limited to two large studies. A study of 1073 Jewish Israeli BRCA carriers, 164 of whom received infertility treatment and 909 of whom did not, demonstrated that fertility treatment regardless of type of medication used was not associated with invasive epithelial ovarian cancer risk [80]. A matched case-control study of 941 pairs of BRCA1 and BRCA2 carriers similarly showed no significant relationship, regardless of type of fertility medication, between both IVF/ IUI and ovarian cancer risk [81].

The literature does suggest that women with infertility in the general population are at higher risk of having ovarian borderline tumors. The first meta-analysis to independently assess the risk of borderline tumors comprised twelve case control studies and showed a four-fold increased risk associated with infertility treatment. These findings have been supported by numerous subsequent studies including a pooled analysis of 8 case-control studies by Ness et al. and a cohort of 19,000 IVF patients by Leeuwen et al [82-85]. Therefore, infertility diagnosed from DOR may be a sign or symptom of underlying, preexisting borderline tumors. Furthermore, the absolute risk of borderline tumors remains low as the baseline risk of borderline tumors is $0.2 \%$ in the general population. [86]. To our knowledge there are no studies specifically assessing the risk of borderline tumors in BRCA patients undergoing $\mathrm{COH}$.

\section{Conclusion}

BRCA carriers are at a higher risk of developing both breast and ovarian cancer, and at a young age, and therefore require vigilant screening to prevent cancer-associated morbidity and mortality. The National Cancer Comprehensive Network recommends a risk-reducing BSO between ages 35 and 40 or after the completion of childbearing. Since many women have not completed childbearing by age 40 , they have the option of undergoing fertility preservation with either oocyte or embryo cryopreservation prior to undergoing this risk-reducing surgery. PGD is a modern technique that may be employed with embryo cryopreservation to assist in the selection of embryos without a BRCA mutation, which could avoid the propagation of BRCA1 and/or BRCA2 to future offspring. Many studies continue to demonstrate ART treatment to be safe in BRCA carriers. Any association to fertility and BRCA carrier status remains conflicting in the reproductive community. Some studies suggest BRCA carriers may have decreased ovarian reserve compared with the general population, an effect that may be more pronounced in carriers of the BRCA1 mutation. However, others have failed to confirm this association.

BRCA carriers are faced with complex challenges and need health care professionals to discuss not only the medical implications of their carrier status and risk-reducing options, but to also share information regarding fertility preservation and the use of PGD to prevent transmission to their offspring. It is important for BRCA carriers to know that there are ways they can safely build a family. Patients are advised to meet with a reproductive specialist as early as possible, especially since advanced maternal age remains a central concern to a patient's chance to achieve a successful pregnancy.

\section{References}

1) Collaborative Group on Hormonal Factors in Breast Cancer. Familial breast cancer: collaborative reanalysis of individual data from 52 epidemiological studies including 58,209 women with breast cancer and 101,986 women without the disease. The Lancet 358:13891399 (2001).

2) Risch HA, McLaughlin JR, Cole DE, Rosen B, Bradley L, Fan I, et al. (2006) Population BRCA1 and BRCA2 mutation frequencies and cancer penetrances: a kin-cohort study in Ontario, Canada. Journal of the National Cancer Institute 98:1694-1706.

3) Robson ME, Boyd J, Borgen PI, Cody HS (2001) Hereditary breast cancer. Current Problems in Surgery. 38:387-480.

4) Rubin SC, Blackwood MA, Bandera C, Behbakht K, Benjamin I, Rebbeck TR, et al. (1998) BRCA1, BRCA2, and hereditary nonpolyposis colorectal cancer gene mutations in an unselected ovarian cancer population: relationship to family history and implications for genetic testing. American Journal of Obstetrics and Gynecology 178:670-677.

5) Whittemore AS, Gong G, Itnyre J (1997) Prevalence and contribution of BRCA1 mutations in breast cancer and ovarian cancer: results from three US population-based case-control studies of ovarian cancer. American Journal of Human Genetics 60:496.

6) Roa BB, Boyd AA, Volcik K, Richards CS (1996) Ashkenazi Jewish population frequencies for common mutations in BRCA1 and BRCA2. Nature Genetics 14:185. 
7) Chen S, Parmigiani G (2007) Meta-analysis of BRCA1 and BRCA2 penetrance. Journal of Clinical Oncology 25:1329-1333.

8) Kuchenbaecker KB, Hopper JL, Barnes DR, Philips KA, MooijTM, et al. (2005) Risks of breast, ovarian, and contralateral breast cancer for BRCA1 and BRCA2 mutation carriers. JAMA 294:1925.

9) Mavaddat N, Peock S, Frost D, Ellis S, Platte R, Fineberg E, et al. (2013) Cancer risks for BRCA1 and BRCA2 mutation carriers: results from prospective analysis of EMBRACE. Journal of the National Cancer Institute 105:812-822.

10) American College of Obstetricians and Gynecologists. ACOG Practice Bulletin No. 103: Hereditary breast and ovarian cancer syndrome. Obstetrics and Gynecology 113:957 (2009).

11) National Comprehensive Cancer Network (2018) Genetic/familial high-risk assessment: breast and ovarian. NCCN clinical practice guidelines in oncology 1 .

12) Warner E, Plewes DB, Hill KA, Causer PA, Zubovits JT, et al. (2004) Surveillance of BRCA1 and BRCA2 mutation carriers with magnetic resonance imaging, ultrasound, mammography, and clinical breast examination. JAMA 292:1317-1325.

13) Kriege M, Brekelmans CT, Boetes C, Besnard PE, Zonderland HM, et al. (2004) Efficacy of MRI and mammography for breastcancer screening in women with a familial or genetic predisposition. New England Journal of Medicine 351:427-437.

14) Skytte AB, Crüger D, Gerster M, Laenkholm AV, Lang C, et al. (2011) Breast cancer after bilateral risk-reducing mastectomy. Clinical Genetics 79:431-437.

15) Nelson HD, Pappas M, Zakher B, Mitchell JP, Okinaka-Hu L, Fu R. (2014) Risk assessment, genetic counseling, and genetic testing for BRCA-related cancer in women: a systematic review to update the US Preventive Services Task Force recommendation. Annals of Internal Medicine 160:255-266.

16) Domchek SM, Friebel TM, Singer CF, Evans DG, Lynch HT, et al. (2010) Association of risk-reducing surgery in BRCA1 or BRCA2 mutation carriers with cancer risk and mortality. JAMA 304:967-975.

17) Davidson BA, Moorman PG (2014) Risk-benefit assessment of the combined oral contraceptive pill in women with a family history of female cancer. Expert opinion on drug safety 13:1375-82.

18) Finch A, Beiner M, Lubinski J, Lynch HT, Moller P, et al. (2006) Salpingo-oophorectomy and the risk of ovarian, fallopian tube, and peritoneal cancers in women with a BRCA1 or BRCA2 Mutation. JAMA 296:185-192.

19) Kindelberger DW, Lee Y, Miron A, Hirsch MS, Feltmate C, Medeiros F, et al. (2007) Intraepithelial carcinoma of the fimbria and pelvic serous carcinoma: evidence for a causal relationship. The American Journal of Surgical Pathology 31:161-169.

20) Kwon JS, Tinker A, Pansegrau G, McAlpine J, Housty M, et al. ( 2013) Prophylactic salpingectomy and delayed oophorectomy as an alternative for BRCA mutation carriers. Obstetrics \& Gynecology 121:14-24.

21) Finch A, Evans G, Narod SA (2012) BRCA carriers, prophylactic salpingo-oophorectomy and menopause: clinical management considerations and recommendations. Women's Health 8:543-55.

22) Practice Committee of the American Society for Reproductive Medicine. Testing and interpreting measures of ovarian reserve: a committee opinion. Fertility and Sterility 98:1407-1415 (2012).

23) Scully R, Livingston DM (2000) In search of the tumour-suppressor functions of BRCA1 and BRCA2. Nature 408:429-432.
24) Jackson SP, Bartek J (2009) The DNA-damage response in human biology and disease. Nature 461:1071-1078.

25) Titus S, Li F, Stobezki R, Akula K, Unsal E, et al. (2013) Impairment of BRCA1-related DNA double-strand break repair leads to ovarian aging in mice and humans. Science Translational Medicine 5:172ra121-172ra121.

26) Jazayeri A, Falck J, Lukas C, Bartek J, Smith GCM, Lukas J, et al. (2006) ATM-and cell cycle-dependent regulation of ATR in response to DNA double-strand breaks. Nature Cell Biology 8:37-45.

27) Tronov VA, Loginova MJ, Kramarenko II (2008) Methylnitrosourea as challenge mutagen in assessment of the DNA mismatch repair (MMR) activity: association with some types of cancer. Russian Journal of Genetics 44:595-600.

28) Xiong B, Li S, Ai J-S, Yin S, et al. (2008) BRCA1 is required for meiotic spindle assembly and spindle assembly checkpoint activation in mouse oocytes. Biology of Reproduction 79:718-726.

29) Sekhon L, Rodriguez-Purata J, Lee J, Whitehouse M (2016) Ovarian reserve and embryonic aneuploidy rates in BRCA 1 and 2 carriers. Fertility and Sterility 106:e371.

30) d'Estaing SG, Perrin D, Lenoir GM, Guérin JF, Dante R (2005) Upregulation of the BRCA1 gene in human germ cells and in preimplantation embryos. Fertility and Sterility 84:785-788.

31) Hakem R, de la Pompa JL, Sirard C, Mo R, Woo M, et al. (1996) The tumor suppressor gene BRCA1 is required for embryonic cellular proliferation in the mouse Cell 85:1009-1023.

32) Gal I, Sadetzki S, Gershoni-Baruch R, Oberman B, Carp H, et al. (2004) Offspring gender ratio and the rate of recurrent spontaneous miscarriages in Jewish women at high risk for breast/ovarian cancer. The American Journal of Human Genetic 74:1270-1275.

33) Lin WT, Beattie M, Chen L-M, Oktay K, Crawford SL, Gold EB, et al. (2013) Comparison of age at natural menopause in BRCA1/2 mutation carriers with a non-clinic-based sample of women in northern California. Cancer 119:1652-1659

34) Finch A, Valentini A, Greenblatt E, Lynch HT, Ghadirian P, et al. (2013) Frequency of premature menopause in women who carry a BRCA1 or BRCA2 mutation. Fertility and Sterility 99:1724-1728.

35) Michaelson-Cohen R, Mor P, Srebnik N, Beller U, Levy-Lahad E, et al. (2014) BRCA mutation carriers do not have compromised ovarian reserve. International Journal of Gynecological Cancer 24:233237.

36) Wang ET, Pisarska MD, Bresee C, Chen Y-DI, Lester J, Afshar $\mathrm{Y}$, et al. (2014) BRCA1 germline mutations may be associated with reduced ovarian reserve. Fertility and Sterility 102:1723-1728.

37) Phillips K-A, Collins IM, Milne RL, McLachlan SA, Friedlander $\mathrm{M}$, et al. (2016) Anti-Müllerian hormone serum concentrations of women with germline BRCA1 or BRCA2 mutations. Human Reproduction dew044.

38) Johnson L, Sammel MD, Domchek S, Schanne A, Prewitt M, et al. (2017) Antimüllerian hormone levels are lower in BRCA2 mutation carriers. Fertility and Sterility 107:1256-65.

39) Oktay K, Kim JY, Barad D, Babayev SN (2010) Association of BRCA1 mutations with occult primary ovarian insufficiency: a possible explanation for the link between infertility and breast/ovarian cancer risks. Journal of Clinical Oncology 28:240-244.

40) Derks-Smeets IA, van Tilborg TC, van Montfoort A, Smits L, Torrance HL, Meijer-Hoogeveen M, et al. (2017) BRCA1 mutation carriers have a lower number of mature oocytes after ovarian stimulation for IVF/PGD. Journal of Assisted Reproduction and Genetics 1-8. 
41) Shapira M, Raanani H, Feldman B, Srebnik N, Dereck-Haim S, Manela D, et al. (2015) BRCA mutation carriers show normal ovarian response in in vitro fertilization cycles. Fertility and Sterility 104:1162-1167.

42) Lin W, Titus S, Moy F, Ginsburg ES, Oktay K (2017) Ovarian Aging in Women With. The Journal of Clinical Endocrinology \& Metabolism 102:3839-47.

43) Ormondroyd E, Donnelly L, Moynihan C, Savona C, et al. (2012) Attitudes to reproductive genetic testing in women who had a positive BRCA test before having children: a qualitative analysis. European Journal of Human Genetics 20:4-10.

44) Schover LR (2009) Patient attitudes toward fertility preservation. Pediatric Blood \&Cancer 53:281-284.

45) Practice Committee of the American Society for Reproductive Medicine, and Practice Committee of the Society for Assisted Reproductive Technology.Ovarian tissue and oocyte cryopreservation. Fertility and Sterility 90:S241-S246 (2008).

46) Oktay K, Cil AP, Bang H (2006) Efficiency of oocyte cryopreservation: a meta-analysis. Fertility and Sterility 86:70-80.

47) (2009) Practice Committee of the American Society for Reproductive Medicine. ASRM Practice Committee response to Rybak and Lieman: elective self-donation of oocytes. Fertility and Sterility 92:1513-1514.

48) Ethics Committee of the American Society for Reproductive Medicine. Fertility preservation and reproduction in patients facing gonadotoxic therapies: a committee opinion. Fertility and Sterility 100:1224-1231 (2013).

49) Wilkinson E (2012) Preimplantation genetic diagnosis for mutated BRCA genes. The Lancet Oncology 13:e331.

50) Kim SS, Klemp J, Fabian C (2011) Breast cancer and fertility preservation. Fertility and Sterility 95:1535-1543.

51) Hickey M, Peate M, Saunders C, Friedlander M (2009) Breast cancer in young women and its impact on reproductive function. Human Reproduction Update 15:323-339.

52) Quinn GP, Vadaparampil ST, Tollins S, Miree CA, Murphy D, et al. (2010) BRCA carriers' thoughts on risk management in relation to preimplantation genetic diagnosis and childbearing: when too many choices are just as difficult as none. Fertility and Sterility 94:2473-5.

53) Kim J, Skrzynia C, Mersereau JE (2015) A pilot study of BRCA mutation carriers' knowledge about the clinical impact of prophylactic-oophorectomy and views on fertility consultation: a single-center pilot study. Journal of Genetic Counseling 24:149-157.

54) Staton AD, Kurian AW, Cobb K, Mills MA, Ford JM (2008) Cancer risk reduction and reproductive concerns in female BRCA1/2 mutation carriers. Familial Cancer 7:179-186.

55) Menon U, Harper J, Sharma A, Fraser L, Burnell M, ElMasry K, et al. (2007) Views of BRCA gene mutation carriers on preimplantation genetic diagnosis as a reproductive option for hereditary breast and ovarian cancer. Human Reproduction 22:1573-1577.

56) Quinn G, Vadaparampil S, Wilson C, King L, Choi J, Miree C, et al. (2009) Attitudes of high-risk women toward preimplantation genetic diagnosis. Fertility and Sterility 91:2361-2368.

57) Strømsvik N, Råheim M, Øyen N, Gjengedal E (2009) Men in the women's world of hereditary breast and ovarian cancer-a systematic review. Familial Cancer 8:221-229.

58) Derks-Smeets IA, Gietel-Habets JJ, Tibben A, Tjan-Heijnen VC, Meijer-Hoogeveen M, et al. (2014) Decision-making on preimplantation genetic diagnosis and prenatal diagnosis: a challenge for couples with hereditary breast and ovarian cancer. Human Reproduction 29:1103-12.
59) Quinn GP, Pal T, Murphy D, Vadaparampil ST, Kumar A (2012) High-risk consumers' perceptions of preimplantation genetic diagnosis for hereditary cancers: a systematic review and meta-analysis. Genetics in medicine 14:191-200.

60) Quinn GP, Vadaparampil ST, Bower B, Friedman S, Keefe DL (2009) Decisions and ethical issues among BRCA carriers and the use of preimplantation genetic diagnosis. Minerva medica 100:371-83.

61) Quinn GP, Vadaparampil ST, King LM, Miree CA, Friedman S (2009) Conflict between values and technology: perceptions of preimplantation genetic diagnosis among women at increased risk for hereditary breast and ovarian cancer. Familial cancer 8:441-9.

62) Gietel-Habets JJ, de Die-Smulders CE, Derks-Smeets IA, Tibben A, et al. (2017) Awareness and attitude regarding reproductive options of persons carrying a BRCA mutation and their partners. Human Reproduction 32:588-97.

63) Brandt AC, Tschirgi ML, Ready KJ, Sun C, Darilek S, Hecht J, et al. (2010) Knowledge, attitudes, and clinical experience of physicians regarding preimplantation genetic diagnosis for hereditary cancer predisposition syndromes. Familial Cancer 9:479-487.

64) Chan J, Johnson L, DiGiovanni L, Voong C, Sammel MD, et al. (2015) Reproductive decision-making in patients diagnosed with BRCA mutations. Fertility and Sterility 104:e76.

65) Goodwin T, Elizabeth Oosterhuis B, Kiernan M, Hudson MM, Dahl GV (2007) Attitudes and practices of pediatric oncology providers regarding fertility issues. Pediatric Blood \&Cancer 48:80-85.

66) Loren AW, Mangu PB, Beck LN, Brennan L, Magdalinski J, Partridge AH, et al. (2013) Fertility preservation for patients with cancer: American Society of Clinical Oncology clinical practice guideline update. Journal of Clinical Oncology 31:2500-2510.

67) Lee SJ, Schover LR, Partridge AH, Patrizio P, Wallace WH, Hagerty K, et al. (2006) American Society of Clinical Oncology recommendations on fertility preservation in cancer patients. Journal of Clinical Oncology 24:2917-2931.

68) Treff NR, Scott RT Jr. (2013) Four-hour quantitative real-time polymerase chain reaction-based comprehensive chromosome screening and accumulating evidence of accuracy, safety, predictive value, and clinical evidence. Fertility and Sterility 99:1049-53.

69) Werner M, Reh A, Grifo J, Perle MA (2012) Characteristics of chromosomal abnormalities diagnosed after spontaneous abortions in an infertile population. Journal of Assisted Reproduction and Genetics 29:817-820.

70) Insogna IG, Ginsburg E (2016) Transferring embryos with indeterminate PGD results: the ethical implications. Fertility Research and Practice 2:2.

71) Zreik TG, Mazloom A, Chen Y, Vannucci M, Pinnix CC, Fulton S, et al. (2010) Fertility drugs and the risk of breast cancer: a metaanalysis and review. Breast Cancer Research and Treatment 124:13-26 72) van den Belt-Dusebout AW, Spaan M, Lambalk CB, Kortman M, Laven JSE, et al. (2016) Ovarian stimulation for in vitro fertilization and long-term risk of breast cancer. JAMA 316:300-312

73) Kotsopoulos J, Librach CL, Lubinski J, Gronwald J, Kim-Sing C, Ghadirian P, et al. (2008) Infertility, treatment of infertility, and the risk of breast cancer among women with BRCA1 and BRCA2 mutations: a case-control study. Cancer Causes \& Control 19:1111-1119.

74) Kim J, Turan V, Oktay k (2016) Long-term safety of letrozole and gonadotropin stimulation for fertility preservation in women with breast cancer.The Journal of Clinical Endocrinology \& Metabolism. 101:1364-1371. 
75) Rizzuto I, Behrens RF, Smith LA (2013) Risk of ovarian cancer in women treated with ovarian stimulating drugs for infertility. The Cochrane Library 8.

76) Lerner-geva L, Geva E, Lessing J, Chetrit A, Modan B, Amit A (2003) The possible association between in vitro fertilization treatments and cancer development. International Journal of Gynecological Cancer 13:23-27.

77) Bamford P, Steele S (1982) Uterine and ovarian carcinoma in a patient receiving gonadotrophin therapy. BJOG: An International Journal of Obstetrics \& Gynaecology 89:962-964.

78) Rossing MA, Daling JR, Weiss NS, Moore DE (1994) Self SG. Ovarian tumors in a cohort of infertile women. New England Journal of Medicine 331:771-776.

79) Tazelaar HD, Bostwick DG, Ballon SC, Hendrickson MR, Kempson RL (1985) Conservative treatment of borderline ovarian tumors. Obstetrics \& Gynecology 66:417-422.

80) Perri T, Lifshitz D, Sadetzki S, Oberman B, Meirow D, Ben-Baruch $G$, et al. (2015) Fertility treatments and invasive epithelial ovarian cancer risk in Jewish Israeli BRCA1 or BRCA2 mutation carriers. Fertility and Sterility 103:1305-1312.

81) Gronwald J, Glass K, Rosen B, Karlan B, Tung N, Neuhausen SL, et al. (2016) Treatment of infertility does not increase the risk of ovarian cancer among women with a BRCA1 or BRCA2 mutation. Fertility and Sterility 105:781-785.

82) Ness RB, Cramer DW, Goodman MT, Kjaer SK, Mallin K, Mosgaard BJ, et al. (2002) Infertility, fertility drugs, and ovarian cancer: a pooled analysis of case-control studies. American Journal of Epidemiology. 155:217-224.

83) Beiner ME, Gotlieb WH, Davidson B, Kopolovic J, Ben-Baruch G (2001) Infertility treatment after conservative management of borderline ovarian tumors. Cancer 92:320-325.

84) Wallach EE, Shoham Z (1994) Epidemiology, etiology, and fertility drugs in ovarian epithelial carcinoma: where are we today? Fertility and Sterility 62:433-448.

85) Van Leeuwen F, Klip H, Mooij T, van de Swaluw AMG, Lambalk CB, Kortman M, et al. (2011) Risk of borderline and invasive ovarian tumours after ovarian stimulation for in vitro fertilization in a large Dutch cohort. Human Reproduction 322.

86) Skirnisdottir I, Garmo H, Wilander E, Holmberg L (2008) Borderline ovarian tumors in Sweden 1960-2005: trends in incidence and age at diagnosis compared to ovarian cancer. International Journal of Cancer 123:1897.

\section{Submit your manuscript to a JScholar journal} and benefit from:

- Convenient online submission

- Rigorous peer review

- Immediate publication on acceptance

ศ Open access: articles freely available online

ฯ High visibility within the field

I Better discount for your subsequent articles

Submit your manuscript at

http://www.jscholaronline.org/submit-manuscript.php 